\title{
La educación superior en Colombia: su alineación con las políticas públicas y la realidad mundial ante el COVID-19
}

\section{Higher Education in Colombia: Its Alignment with Public Policies and the World Reality in the face of COVID-19}

Deybi Yolima Jiménez-González;

María Clemencia Molina-Parra²

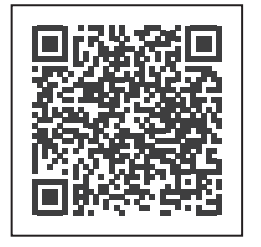

Palabras clave: calidad de la educación, cambio social, COVID-19, economía solidaria, enseñanza superior.

Artículo de revisión

Fecha de recepción: 30/10/2020

Fecha de aprobación: 24/05/2021

Esta publicación se encuentra bajo licencia:

Creative Commons

Reconocimiento-

NoComercial-

SinObraDerivada 4.0

Internacional

\section{Resumen}

Problemática: el dinamismo que genera la globalización y los cambios que propone el contexto mundial son un llamado a la reestructuración general de todos los escenarios y especialmente el relacionado con la educación superior en Colombia. Objetivo: por lo tanto, es necesario conocer el contexto de la educación superior en Colombia para comprender la alineación con las políticas que proponen los gobiernos y las orientaciones que surgen de la realidad mundial en tiempos de pandemia. Materiales y métodos: así, con una investigación exploratoria, se llevó a cabo el análisis sistemático de la literatura para consolidar las últimas directrices de las autoridades en el sector educativo, teniendo en cuenta el rápido desarrollo y la extensión temática en el campo de estudio. Resultados: en la actualidad, se han implementado otros modelos educativos diferentes al tradicional (presencial), los cuales incluyen el uso de portales de conocimiento, redes sociales y plataformas colaborativas que aprovechan los múltiples beneficios que ofrecen las TIC y donde la

1 Administración de Empresas en formación. Estudiante, Universidad Cooperativa de Colombia, Colombia. deybi.jimenezg@campusucc.edu.co, ORCID: 0000-0002-0340-4380

2 Economista, MBA, Tecnológico de Monterrey, México. Universidad Cooperativa de Colombia, Colombia. maria.molina@ucc.edu.co, ORCID: 0000-00030150-4369 


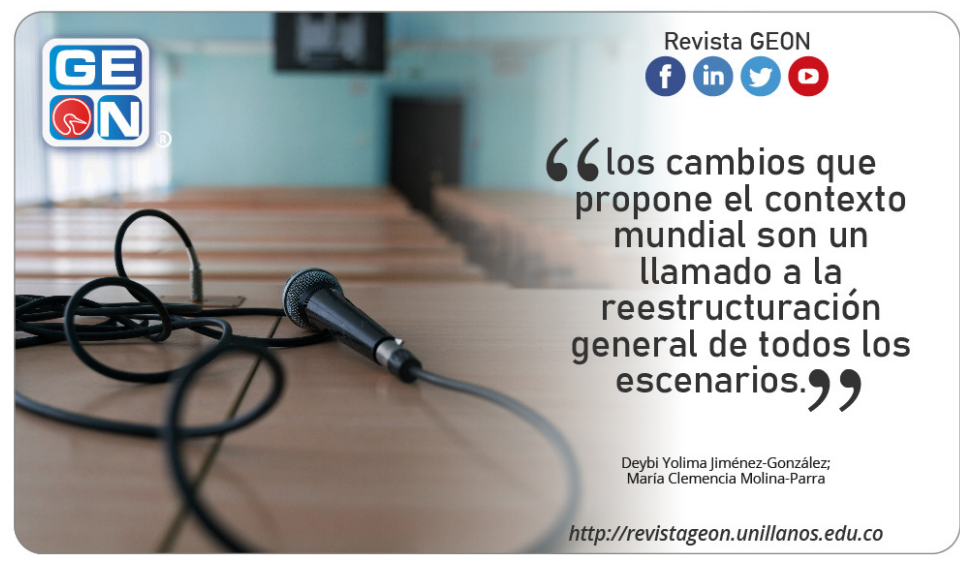

metodología Blended Learning ha ganado mayor protagonismo por su capacidad de combinar, aprovechar y complementar los elementos que aportan dos entornos diferentes (físico y virtual). Discusión: de esta manera, en línea con las nuevas exigencias que impone el mercado laboral, se requiere de una educación para la vida que involucre el desarrollo de soft skills, que tengan en cuenta que las habilidades blandas son cada vez más necesarias para estar a la vanguardia y responder a las exigencias del contexto. Conclusión: puesto que la realidad que afronta el mundo ha acelerado todos los procesos de transformación y ha hecho que se produzcan cambios más rápidos y de forma continua, paradójicamente esto se ha convertido en una oportunidad para conseguir avances significativos en educación a nivel mundial.

Palabras claves: calidad de la educación, cambio social, COVID-19, economía solidaria, enseñanza superior.

Cómo citar este artículo / Toreference this article:

Jiménez González, D. Y., \& Molina Parra, M. C. (2021). La educación superior en Colombia: su alineación con las políticas públicas y la realidad mundial ante el COVID-19. Revista GEON (Gestión, Organizaciones Y Negocios), 8(1), e-290. https://doi. org/10.22579/23463910.290
Códigos JEL: 120, 121, O30, Q50

\section{Abstract}

Problematic: The dynamism generated by globalization and the changes proposed by the world context are a call for the general restructuring of all scenarios and especially that related to higher education in Colombia. Objective: Therefore, it is necessary to know the context of higher education in 
Cómo citar este artículo / Toreference this article:

Jiménez González, D. Y., \& Molina Parra, M. C. (2021). La educación superior en Colombia: su alineación con las políticas públicas y la realidad mundial ante el COVID-19. Revista GEON (Gestión, Organizaciones Y Negocios), 8(1), e-290. https://doi. org/10.22579/23463910.290
Colombia to understand the alignment with the policies proposed by the governments and the orientations that arise from the world reality in times of pandemic. Materials and methods: Thus, with an exploratory research, a systematic literature analysis was carried out to consolidate the latest guidelines from the authorities in the education sector, considering the rapid development and thematic extension in the field of study. Results: Currently, other educational models different from the traditional one (face-to-face) have been implemented, which include the use of knowledge portals, social networ$\mathrm{ks}$, and collaborative platforms, taking advantage of the multiple benefits offered by ICT's and where the Blended Learning methodology has gained greater prominence due to its ability to combine, take advantage of and complement the elements provided by two different environments (physical and virtual).

Discussion: In this way, in line with the new demands imposed by the labor market, an education for life is required that involves the development of Soft Skills, considering that soft skills are increasingly necessary to be at the forefront and respond to the demands of the context. Conclusion: Since the reality facing the world has accelerated all transformation processes, causing changes to take place more rapidly and continuously, paradoxically this has become an opportunity to achieve significant advances in education worldwide.

Keywords: COVID-19; Educational quality; Higher education; Social change; Solidarity economy.

\section{Introducción}

El dinamismo generado por la globalización y los cambios propuestos por el entorno mundial llevan a que la investigación busque explicar cuál es el contexto de la educación superior en Colombia, su alineación con las políticas gubernamentales y las orienta- ciones académicas, partiendo de la existencia de un importante volumen de información primaria, el rápido desarrollo y la extensión temática en este campo. Por lo tanto, es fundamental consolidar las últimas directrices de las autoridades y los entes gubernamentales al reconocer los cambios que enfrenta la educación 
superior en Colombia en tiempos de emergencia sanitaria, tales como el uso de la metodología Blended Learning, la utilización de otros modelos educativos diferentes al tradicional y los múltiples desafíos que tienen los gobiernos y las instituciones de educación superior en relación con el cierre de la brecha digital.

A continuación, el contexto teórico presenta, en términos generales, la esencia de la educación como derecho fundamental e instrumento esencial para garantizar las condiciones básicas de una vida digna, resaltando los factores que han marcado la educación en América Latina y el Caribe, junto con los requerimientos que exige un cambio de modelo pedagógico. La sección de materiales y métodos describe el uso de la investigación exploratoria como herramienta utilizada para indagar y consolidar, a través del análisis sistemático de literatura, el estado de la educación superior en Colombia, su alineación con las políticas públicas y la realidad mundial. Seguido, se exponen los resultados con los principales cambios que se han presentado en las dinámicas de aprendizaje tras la pandemia del COVID-19 en marzo de 2020, la promoción de un modelo de alternancia como opción a la suspensión de las clases presenciales y la influencia de la metodología Blended Learning para articular dos modalidades diferentes de enseñanza y aprendizaje, además de los lineamientos en materia de educación superior de acuerdo con las políticas públicas nacionales, departamentales y locales y su relación con la economía social y solidaria.
Luego, la discusión presenta la comparación entre los resultados descritos anteriormente y las nuevas exigencias que impone el mercado laboral según la perspectiva del gerente de desarrollo de negocios para Microsoft, Pedro Uribe, quien explica el uso de la tecnología como medio de comunicación, de relación y de aprendizaje y las actuaciones del gobierno colombiano en pro de generar las condiciones para continuar con el proceso de formación bajo la modalidad de trabajo académico en casa y posteriormente el retorno gradual y progresivo a las instituciones. Por otra parte, las conclusiones hacen énfasis en el reto tecnológico que tiene Colombia en cuanto al equipamiento y acceso a las plataformas digitales en el contexto educativo para lograr, a través de los próximos planes de desarrollo, que la tecnología sea el principal aliado y socio estratégico, capacitando y reconociendo el papel fundamental de los profesores en este nuevo escenario. Finalmente, las referencias permiten evidenciar y comprobar las fuentes utilizadas para argumentar la información presentada en el contenido de este documento.

\section{Contexto teórico}

A nivel mundial, la Organización de las Naciones Unidas para la Educación, la Ciencia y la Cultura (Unesco, 2019c) define la educación como "[...] un derecho humano para todos, durante toda la vida, y donde el acceso debe ir acompañado de calidad" (párr. 2); por lo tanto, al considerarse un derecho fundamental, se convierte en un instrumento esencial que garantiza 
las condiciones básicas para una vida digna. De esta manera, según lo expresa Turbay (2000), "[...] la educación en todas sus manifestaciones es la vía por excelencia de la socialización humana" (p. 9), ya que a partir de este proceso se impactan positivamente los diferentes contextos del entorno. Por ende, es un deber de los gobiernos asegurar la enseñanza en todos los niveles y dimensiones a través de las instituciones de enseñanza y educación superior (Unesco, 2019a), lo que implica para los gobiernos un reto enorme en el proceso de contribución al pleno desarrollo de la sociedad, de modo que las políticas públicas en educación deben ir sustentadas con una mayor destinación de recursos económicos y de capital intelectual.

Según Didriksson (2008), en el contexto de América Latina y el Caribe, la educación ha estado envuelta en un rezago científico y tecnológico marcado principalmente por la exclusión y la desigualdad en el gasto público en educación (CEPALSTAT, 2019), además de los vacíos que dejan las políticas públicas emitidas por cada país y que en conjunto acrecientan la brecha de la deuda social en educación (Barcena et al., 2017).

En este sentido, la educación requiere de una transformación donde las instituciones de educación superior sean los principales motores y actores del proceso de cambio, y en conjunto con el gobierno, la comunidad y los demás actores clave articulen las estrategias "[...] para producir y transferir conocimientos científico-tecnológico a nivel nacional, regional e internacional" (Didriksson, 2012, p. 193). Por lo anterior, dicho autor hace énfasis en "[...] un cambio de modelo pedagógico y organizacional que comprende que la acción educativa se sostiene en la unidad de lo diferente" (p. 193); es decir, en su capacidad de crear o contribuir a la identidad de los ciudadanos a partir de una metodología que permee para la vida.

\section{Materiales y métodos}

Teniendo en cuenta el rápido desarrollo y la extensión temática en el campo de la educación superior relacionada con la existencia de un importante volumen de información primaria, se decide realizar un análisis sistemático de la literatura para consolidar las últimas directrices de las autoridades en el sector educativo. Para esto, se recurre a fuentes digitales de organizaciones nacionales e internacionales para argumentar el desarrollo de la investigación a partir de la utilización de los contenidos que suministra la Unesco, la Comisión Económica para América Latina y el Caribe (CEPAL), el Ministerio de Educación Nacional, el Ministerio de Tecnologías de la Información y las Comunicaciones, el Plan de Desarrollo Nacional, Departamental y Municipal, entre otros.

Se comenzó con un tipo de investigación exploratoria con el fin de establecer un estado del arte que permitiera indagar sobre los efectos ocasionados por el COVID-19 en la educación superior y los lineamientos trazados desde la Unesco con sus orientaciones académicas, que guían el camino de las instituciones de edu- 
cación para superar la crisis. Así, se planteó un análisis de literatura conjugando documentos de la CEPAL y del Ministerio de Educación Nacional para entrar a explicar el contexto de la educación superior y su alineación con las políticas gubernamentales.

\section{Resultados}

En el contexto pedagógico, la educación superior a nivel mundial se rige bajo los lineamientos que indica la Unesco (2019b), por tratarse de un derecho humano fundamental al que pueden tener accesos todos los seres humanos bajo plenas garantías y condiciones de calidad. Sin embargo, debido a la pandemia del COVID-19 en marzo de 2020 decretada por la Organización Mundial de la Salud (OMS), las dinámicas de aprendizaje fueron permeadas por esta y obligaron al cierre masivo y a la suspensión de las clases presenciales en más de 190 países. Por tanto, desde entonces se han implementado otros modelos educativos diferentes al tradicional, entre ellos la educación mediada por la tecnología o el "[...] despliegue de modalidades de aprendizaje a distancia, mediante la utilización de una diversidad de formatos y plataformas (con o sin uso de tecnología)" (CEPAL-Unesco, 2020, p. 1), de forma que se han tenido que "[...] sustituir los encuentros en aulas por clases en línea" (Cardona Montoya, 2020). Dado lo anterior, se entiende que el "[...] aprendizaje en esta época es un proceso continuo en ambientes discontinuos: el aula, la biblioteca, el campus virtual, las plataformas de comunicación digital, las redes sociales, la empresa, el laboratorio, etc." (Cardona Montoya, 2020), que ayudan para que el proceso formativo no se detenga y el sistema educativo sea flexible ante la realidad que afronta el mundo.

Por tal motivo, se promueve un modelo de alternancia como alternativa a la suspensión de las clases presenciales, las cuales han impuesto desafíos muy significativos para todos los gobiernos $y$, desde luego, para las instituciones de educación superior, quienes finalmente debieron afrontar la continuidad del proceso educativo, en especial en lo relacionado con el acceso a los recursos y las plataformas digitales, medios necesarios para lograr la interacción con el alumno y generar continuidad en el proceso de formación y aprendizaje. De esta forma, "[...] la pandemia del Covid-19 exacerbó las desigualdades sociales, la inequidad y la exclusión, al tiempo que se transformó paradójicamente en una oportunidad" (CEPAL-Unesco, 2020, p. 16) para cambiar los contenidos, las metodologías y los modelos educativos para convertir al alumno en actor primordial de la nueva realidad.

Lo anterior es la esencia que propone la CEPAL-Unesco (2020) acerca de la "[...] definición de educación para la ciudadanía mundial, la cual busca empoderar individualmente a las personas, de tal forma que se apropien y construyan sus realidades sobre la base del fortalecimiento de las relaciones sociales" (p. 17), lo cual ratifica que, sin duda alguna, la respuesta a la crisis ha acelerado los progresos en la enseñanza digital y a distancia de una forma nunca vista, lo que 
cambiará radicalmente las formas de aprendizaje al tornarlas en espacios de colaboración y autoaprendizaje (Echazarra, 2020).

De esta forma, la metodología Blended Learning llegó para quedarse como una de las más influyentes del siglo XXI. El Ministerio de Tecnologías de la Información y las Comunicaciones en Colombia (2020) hace referencia a esta como la flexibilización de la educación usando la combinación de metodologías semipresenciales y plataformas electrónicas como medios de formación para dar soporte a los procesos de enseñanza y aprendizaje.

Según Inspira TICs (2020), la aplicación de la metodología busca combinar, aprovechar y complementar todos los elementos que aporta el entorno físico y virtual para articular dos modalidades diferentes de enseñanza y aprendizaje, con el objetivo de que allí se puedan desarrollar varias metodologías pedagógicas que permitan enriquecer el proceso de formación de los alumnos. Dicho esto, la metodología Blended Learning trae consigo un mayor grado de flexibilidad, puesto que permite adaptar el horario y la disponibilidad de información de acuerdo con la programación de los encuentros, los cuales pueden ser presenciales o virtuales, sincrónicos o asincrónicos, por lo que permite a todos los actores que intervienen en el proceso aumentar la competencia digital a través de la práctica y el uso de recursos y herramientas en línea. Alli "[...] el profesor cumple dos roles; como tutor presencial y como tutor on-line y el estudiante se convierte en el centro del aprendizaje" (Abadía Alvarado, 2016, p. 29).

Ahora bien, al hacer la revisión de las políticas nacionales, teniendo en cuenta que estas fueron trazadas antes de presentarse la emergencia sanitaria, el Departamento Nacional de Planeación (2019), como ente encargado de coordinar la formulación del Plan Nacional de Desarrollo 20182022 Pacto por Colombia, pacto por la equidad, trazó el camino para lograr la legalidad, el emprendimiento y un futuro con igualdad para todos los colombianos. En lo que refiere a la política social moderna centrada en la familia, eficiente, de calidad y conectada a los mercados, esta apunta a la consecución de once objetivos de desarrollo sostenible, entre ellos la educación de calidad. Allí, el gobierno nacional propone una educación de calidad desde el primer nivel de formación con el fin de robustecer el sistema educativo para que este sea capaz de responder a las dinámicas fundamentales que permitan lograr el desarrollo integral de las personas y, por ende, el crecimiento del país; teniendo en cuenta que la educación es el pilar para un futuro con igualdad de oportunidades.

En concordancia con las líneas nacionales, está el Plan de Desarrollo Económico y Social Departamental "Hagamos Grande al Meta" para el período 20202023 (Asamblea Departamental del Meta, 2020, p. 1), en el cual la educación es vista como "[...] uno de los más importantes requisitos para el desarrollo social y económico" (p. 142) de la región. Por lo tanto, en el 
rubro de la educación, se puede destacar el accionar de la administración departamental en pro de lograr mejores condiciones en el proceso de enseñanza y aprendizaje, de manera que se logre un impacto real y efectivo en el entorno local.

A nivel municipal, el Plan de Desarrollo "Villavicencio Cambia Contigo" 20202023 tiene como objetivo fundamental "[...] avanzar en el propósito de hacer de Villavicencio una ciudad moderna, incluyente y participativa, bajo los pilares del arraigo y la construcción de identidad con el territorio, la dignidad humana y la transformación urbana hacia una ciudad moderna" (Secretaría de Planeación de Municipal, 2020, p. 8). Dado lo anterior, para el sector educativo se propone mejorar la calidad, la cobertura y la reducción de las brechas sociales para los grupos más vulnerables, partiendo desde el punto de vista donde el sector educativo es "[...] un componente netamente social" (Secretaría de Planeación de Municipal, 2020, p. 224).

Finalmente, es importante mencionar la relación de la educación superior con la economía social y solidaria debido al amplio protagonismo que ha venido ganando en la agenda pública de los gobiernos, puesto que ha demostrado su convergencia como una alternativa para dar solución a las problemáticas sociales, económicas y ambientales en los territorios. De esta manera, según Pérez Muñoz et al. (2019), se deben aunar esfuerzos desde todas las instituciones que velan e imparten educación superior en Colombia, con el objetivo de empo- derar a la ciudadanía para que pueda crear soluciones sostenibles para el beneficio común y así poder actuar ante los problemas que nos han afectado por décadas, por ejemplo, la pobreza, el calentamiento global y la desigualdad social, entre otros.

\section{Discusión}

Desde los lineamientos trazados por la Unesco se reconoce que la educación es un derecho humano fundamental para todos; por lo tanto, se propone una educación para la vida que involucre el desarrollo de soft skills en línea con las nuevas exigencias que impone el mercado laboral (inteligencia artificial y quinta revolución industrial), según lo comentado por el gerente de desarrollo de negocios para Microsoft, Pedro Julio Uribe, en el Canal UDES (2020a) durante el conversatorio de transformación educativa en un mundo digital, donde las habilidades blandas son cada vez más necesarias para estar a la vanguardia y responder a las exigencias del contexto. De esta manera, de acuerdo con lo expresado por el profesor de Psicología Duart Montoliu en el Canal UDES (2020b), la pandemia que enfrenta el mundo rebasó todos los contextos, sin olvidar que la tecnología llegó para cambiar, transformar y moldear no solo los patrones de vida del ser humano, sino las formas de comunicar, relacionarse y aprender.

En consecuencia, en Colombia, partiendo de que la educación es un servicio público (Constitución Política de Colombia, 1991) y que el gobierno nacional es el responsable de brindar las 
garantías para ello, la presidencia de la República emitió una serie de resoluciones y decretos en pro de generar las condiciones para dar continuidad al proceso de formación bajo la "[...] modalidad de trabajo académico en casa y posteriormente el retorno gradual y progresivo a las instituciones educativas bajo el esquema de alternancia" (Ministerio de Salud y Protección Social de Colombia, 2020, p. 11). En este sentido, desde el Ministerio de Educación Nacional (2020) se dictan medidas, a través de la directiva ministerial n. ${ }^{\circ} 4$ para que las instituciones de educación superior y aquellas autorizadas para ofrecer y desarrollar programas académicos hagan uso de las tecnologías para el desarrollo de los programas presenciales; sin embargo, no todos los estudiantes cuentan con las herramientas tecnológicas para continuar el proceso.

Efectivamente, en todos los escenarios de la vida humana, existe un antes y un después de la pandemia; por lo tanto, la educación superior en Colombia y en el mundo no puede ser indiferente ante esta nueva realidad, situación que condujo a que, "sin experiencia" en la mayoría del país, se optara por modelos educativos diferentes al tradicional, haciendo uso de un sinnúmero de herramientas que pudieran garantizar en cierto grado la continuidad de la educación en todos los niveles como, por ejemplo, la radio, la televisión, las guías digitales y la internet con sus diversas plataformas y aplicaciones, las cuales permiten y facilitan un acercamiento en tiempo sincrónico y asincrónico entre docentes y estudiantes.
De igual manera, en el mismo sentido en el que se reconoce la importancia de la tecnología en esta nueva realidad, también debe quedar expuesta la desigualdad social entre quienes tienen los recursos físicos y de conectividad para continuar con el proceso y quienes carecen de ellos; un ejemplo claro es los estudiantes que viven en el campo y la ciudad bajo condiciones de pobreza.

De esta forma, se evidencian desafíos muy significativos para los gobiernos y las instituciones de educación superior en relación con el equipamiento y el acceso de las plataformas digitales, puesto que, en este sentido, la Comisión Económica para América Latina y el Caribe (CEPAL), con base en los datos de la Organización de Cooperación y Desarrollo Económicos y el Programa para la Evaluación Internacional de Estudiantes (PISA-2020), en el cual Ikeda indicó en el informe emitido en septiembre de 2020 que el "[...] 62\% de los estudiantes de 15 años en Colombia tienen acceso a una computadora; pero solo el $29 \%$ puede acceder a un software educativo" (CEPAL-Unesco, 2020, p. 6). Por lo tanto, para Giannini (2020), el trabajo se centra en lograr el cierre de la brecha digital para que cada vez más jóvenes tengan igualdad de acceso a la educación y además se garantice una conexión a internet de calidad en todo el territorio.

En términos generales, las líneas de acción de los planes de desarrollo están orientadas a ofrecer educación de calidad, mejorar las condiciones de enseñanza, la cobertura y acceso. De 
esta forma, se puede decir que la educación siempre ha estado en un cambio paulatino; sin embargo, lo que ha generado el COVID-19 es acelerar todos los procesos de transformación, haciendo que los cambios se produzcan mucho más rápido y de forma continua. En consecuencia, teniendo en cuenta las transformaciones que está experimentando la sociedad en general, muy seguramente las políticas públicas, los planes y las estrategias entorno a la educación tendrán un enfoque mucho más humano y digital; así, los próximos planes de desarrollo serán diseñados entorno a los desafíos y retos que tiene el país para aprovechar los escenarios posibles que ofrece esta nueva realidad, donde el principal aliado y socio estratégico es la tecnología.

Con lo anterior, se evidencia la necesidad para que desde el gobierno se inste a trabajar arduamente por el bienestar colectivo de las personas y no solo por los intereses de unos pocos. Es imperativo actuar bajo los valores universales que rigen la economía social y solidaria, no solo para abordar los problemas que nos han afectado por décadas, sino para empoderar y construir un país con oportunidades de educación para todos.

\section{Conclusiones}

Si bien es cierto que la educación siempre ha estado en un desarrollo paulatino, el contexto actual ha acelerado todos los procesos de transformación y producido cambios más rápidos y de forma continua, por lo que esto se convierte en un llamado a la reestructuración general de todos los escenarios de la sociedad y especialmente el relacionado con la educación (Ley 30 de 1992; Microdatos - DANE, 2018).

En tiempos de crisis e incertidumbre, el mundo exige que los profesionales líderes, creativos e innovadores estén convocados a actuar desde un enfoque emocional y en equilibrio con el medio ambiente, con el objetivo de impulsar soluciones colectivas a los desafíos urgentes que presenta el mundo y contribuir a su transformación estructural, no solo desde el punto de vista conceptual o técnico, sino desde el ser, la cooperación y las relaciones sociales y solidarias.

Por otro lado, debido a los cambios transcendentales que está experimentando la sociedad en general, muy seguramente las políticas, los planes y las estrategias entorno a la educación superior en Colombia (Decreto 1075, 2015; Decreto 1330, 2019) tendrán un enfoque mucho más digital; por lo tanto, los próximos planes de desarrollo serán diseñados con base en los desafíos y retos que tiene el país en materia de lograr aprovechar todos los escenarios posibles que ofrece esta nueva realidad, donde el principal aliado y socio estratégico es la tecnología.

Ahora bien, no se puede desconocer el papel fundamental de los profesores en este nuevo escenario, puesto que es ahora cuando la pedagogía que caracteriza la forma de enseñanza particular de cada uno toma relevancia en las llamadas competencias 
blandas, aquellas que humanizan la educación e impregnan en el alumno una actitud emocional para convertirse en un actor activo del proceso, con participación proactiva y constructiva en la transformación de la sociedad.

\section{Información complementaria}

Este documento se elaboró como parte del producto final del trabajo de grado para optar al título profesional de Administrador de Empresas en la Universidad Cooperativa de Colombia y se presentó a través de una ponencia para los asistentes al Congreso Internacional Académico y de Investigación: "Contextos organizacionales en tiempos de crisis 2020", organizado por la RedGESTIO. Finalmente, un sincero agradecimiento por el apoyo oportuno e incondicional a la Dra. María Clemencia Molina Parra, quien es coautora en el documento.

\section{Referencias}

Abadía Alvarado, L. K. (2016). Lineamientos con Condiciones Específicas de Calidad para los Programas Ofrecidos en las Modalidades Virtual Combinada (Blended Learning). Oficina de la Innovación Educativa con el Uso de Nuevas Tecnologías.

Asamblea Departamental del Meta. (30 de mayo de 2020). Ordenanza $N^{\circ}$. 1069 de 2020. Por medio de la cual se aprueba y adopta el Plan de Desarrollo Económico y Social Departamental "Hagamos Grande al Meta" para el periodo 2020-2023 y se dictan otras disposiciones. https://bit.ly/3×23Ts1

Barcena, A., Prado, A., Abramo, L. \& Pérez, R. (2017). Fortalecer las políticas sociales a lo largo del ciclo de vida: un enfoque en la infancia, la adolescencia y la juventud. En Brechas, ejes y desafíos en el vínculo entre lo social y lo productivo (pp. 39-82). Naciones Unidas. https://bit.ly/3uZmuns

Canal UDES. (2020a, 20 de octubre). Conversatorio transformación educativa en un mundo digital [video]. YouTube. https://bit.ly/3wogMmv

Canal UDES. (2020b, 24 de septiembre). Conversatorio Destejer un arcoiris: ¿es el blended learning un desafí tecnológico? [video]. YouTube. https://bit.ly/3w09ejv

Cardona Montoya, G. (2020, 13 de mayo). Educación: ni presencial ni virtual, blended (híbrida). La caja registradora. https://bit.ly/3cnCw43

CEPALSTAT. (2019). Gasto público en educación. CEPAL - Naciones Unidas. https://bit.ly/2RPMkwD

Comisión Económica para América Latina y el Caribe, Organización de las Naciones Unidas para la Educación, la Ciencia y la Cultura . (2020). Informe Covid-19: la educación en tiempos de pandemia. https://bit.ly/3pv8T61

Congreso de la República. (1992, 28 de diciembre). Ley 30. Por el cual se organiza el servicio público de la Educación Superior. Diario Oficial n. ${ }^{\circ}$ 40.700. https://bit.ly/3giMd6c

Constitución Política de Colombia. (1991). Gaceta Constitucional n.o 116. http:// bit.ly/2NA2BRg

Departamento Nacional de Planeación. (2019). Bases del Plan Nacional de Desarrollo 2018-2022: Pacto por Colombia, pacto por la equidad. Grupo de Comunicaciones. https://bit.ly/3z6N7tL

Didriksson, A. (2008). Contexto global y regional de la educación superior en 
América Latina y el Caribe. En Tendencias de la Educación Superior en América Latina y el Caribe (pp. 19-52). IESALC-UNESCO. https://bit.ly/3fVtihr

Didriksson, A. (2012). La nueva agenda de transformación de la educación superior en América Latina. Perfiles Educativos. XXXIV(138), 184-203. https:// bit.ly/3g1 $1 \times r 3 i$

Echazarra, A. (2020). Do students learn in co-operative or competitive environments? PISA in FocUs, n.o 7, OECD Publishing. https://doi.org/10.1787/ e7a5f3e4-en

Giannini, S. (2020, 18 de junio). Reconstruir mejor: tras el COVID-19, la educación debe cambiar para responder a la crisis climática. Unesco. https://bit. ly/3gdAYdX

Ikeda, M. (2020). Were schools equipped to teach - and were students ready to learn - remotely? PISA in Focus, n.o 8, OECD Publishing. https://doi.org/10.1787/4bcd7938-en

InspiraTICs. (2020). Blended Learning. un aprendizaje eficiente, por favor; mezclado, no agitado. https://bit.ly/3w4MCyr

Microdatos - DANE. (2018, 29 de julio). Colombia - Educación Formal - 2018. Archivo Nacional de Datos. https://bit. ly/3gikZMP

Ministerio de Educación Nacional. (2020, 22 de marzo). Directiva Ministerial n. ${ }^{\circ}$ 04. Uso de tecnologías en el desarrollo de programas académicos presenciales. https://bit.ly/3gibZHB

Ministerio de Salud y Protección Social de Colombia, Ministerio de Eduación Nacional. (2020, junio). Lineamientos para la prestación del servicio de educación en casa y en presencialidad bajo el esquema de alternancia y la implemen- tación de prácticas de bioseguridad en la comunidad educativa. https://bit. ly/3pGhLGh

Ministerio de Tecnologías de la Información y las Comunicaciones. (2020, 7 de septiembre). B-Learning. https://bit. ly/3zb9DBS

Organización de las Naciones Unidas para la Educación, la Ciencia y la Cultura. (2019a). Derecho a la Educación - Obligaciones y responsabilidades del Estado. https://bit.ly/3clt9l5

Organización de las Naciones Unidas para la Educación, la Ciencia y la Cultura. (2019b). Educación superior y Objetivos de Desarrollo Sostenible. https:// bit.ly/3z5LJHQ

Organización de las Naciones Unidas para la Educación, la Ciencia y la Cultura. (2019c). Education transformd lives. https://bit.ly/3cp6QLn

Organización Mundial de la Salud. (2020). Brote de enfermedad por coronavirus (COVID-19). https://bit.ly/3cA4vxy

Pérez Muñoz, C., Gómez, M. E., Hernández Arteaga, I. \& García Porras, S. (2019). Educación superior y economía solidaria hacia un enfoque territorial. Sophia, 15(1), 16-30. http://dx.doi. org/10.18634/sophiaj.15v.1i.902

Presidencia de la República. (2015, 26 de mayo). Decreto 1075. Por medio del cual se expide el Decreto Único Reglamentario del Sector Educación. Diario Oficial 49523. https://bit.ly/3zxBXP4

Presidencia de la República. (2019, 25 de julio). Decreto 1330. Por el cual se sustituye el Capítulo 2 y se suprime el Capítu107 del Título 3 de la Parte 5 del Libro 2 del Decreto 1075 de 2015 - Único Reglamentario del Sector Educación. https:// bit.ly/3vkDXGO 
Secretaría de Planeación de Municipal. (2020). Plan de Desarrollo Villavicencio Cambia Contigo 2020-2023. https:// bit.ly/3z8KA2f

Turbay, C. (2000). El Derecho a la educación. Desde el marco de la protección integral de los derechos de la niñez y de la política educativa. Unicef Colombia - Fundación Antonio Restrepo Barco. https://uni.cf/3pCiAQk 